\title{
Amine Regeneration Tests on MEA, DEA and MMEA with Respect to Energy Efficiency Study
}

\author{
Yun-Long ZHOU ${ }^{1, a}$, Lin-Na ZHENG ${ }^{1, b}$, Yi-Fan CAO ${ }^{1, c}$, Lu HUANG ${ }^{1, d}$, Huan- \\ Cong $\mathrm{SHI}^{1, \mathrm{e}}$ and Li-feng CUI $\mathrm{CH}^{1, \mathrm{f},{ }^{\prime}}$
}

${ }^{1}$ Department of Environmental Science and Engineering, University of Shanghai for Science and Technology, Shanghai, 200093, P. R. China.

\author{
aalbert0904@163.com, ${ }^{\mathrm{b}} 1012873444 @ q q . c o m,{ }^{\mathrm{c}}$ \\ 645232392@qq.com, d1919645050@qq.com, êhcshi@usst.edu.cn \\ * lifeng.cui@gmail.com
}

Keywords: $\mathrm{CO}_{2}$ desorption, Amine regeneration curves, primary and secondary amines, Heat duty reduction, Energy efficient method

\begin{abstract}
The $\mathrm{CO}_{2}$ desorption analyses of amines were performed to reveal the behaviour of amine regeneration process. A typical primary amine (MEA) and two other secondary amines (MMEA and DEA) were selected in preparation for amine solutions under different concentrations, from 1-7 $\mathrm{mol} / \mathrm{L}$. The regeneration curves were plotted to describe the process. It was discovered that the specific $\mathrm{CO}_{2}$ loading (mol/mol) that distinguish the amine regeneration curves into different regions were the same for the specific amine, despite different concentrations. These points were defined as "turning points" on regeneration curves. The turning points of MEA, MMEA and DEA are located at $\mathrm{CO}_{2}$ loading of $0.40 \mathrm{~mol} / \mathrm{mol}, 0.38 \mathrm{~mol} / \mathrm{mol}$ and $0.28 \mathrm{~mol} / \mathrm{mol}$, respectively. The regeneration tests compared the relative heat duty at the first 2 hours: MMEA > MEA > > DEA.
\end{abstract}

\section{Introduction}

The post combustion carbon dioxide $\left(\mathrm{CO}_{2}\right)$ capture technologies with amine based reactive solvents have been reported for years [1,2].The major research challenge of the conventional amine scrubbing process is to develop some energy efficient methods for heat duty reduction [3]. For amine-based post-combustion $\mathrm{CO}_{2}$ capture (PCCC), close to $70 \%$ of the overall energy is distributed during amine regeneration for $\mathrm{CO}_{2}$ desorption [4]. The heat cost is considered as reboiler heat duty, since the energy required for amine regeneration is supported by hot steam flowing into a reboiler located at the bottom of a regeneration column[5]. This heat duty has been an important parameter for design, operation and development of a cost-effective $\mathrm{CO}_{2}$ absorption-desorption process [4]. According to Rochelle [3], the theoretical minimum energy requirement of $\mathrm{CO}_{2}$ separation along with the $\mathrm{CO}_{2}$ compression is estimated to be $0.11 \mathrm{MWh} /$ tonne $\mathrm{CO}_{2} / 435.6 \mathrm{~kJ} / \mathrm{kg}$ $\mathrm{CO}_{2}$, which remains a challenge to achieve.

A recent published review [6] introduced the comprehensive amine regeneration researches, including four parts. They are 1) conventional heating processes, 2) newly developed methods, 3) optimization of operation parameters and 4) regeneration costs. The last part is very important as the research focus for years. Peer researchers have developed several methods to reduce the part or overall of heat duty[3,4,6-15]. Two conventional methods are recommended: solvent improvement and process intensification[2]. For process configuration optimization, a series of approaches have been conducted since 2005 [2,7,8,9], which focused on the utilization of as much external energy as possible.

This research focused on another direction: solvent improvement [3], which has been investigated repeatedly with heat duty analyses since 2005[4,5,10-15]. The heat duty calculation was conducted [5] based on the $\mathrm{CO}_{2}$ desorbed out of the regeneration column and the amount of lean amine solutions returned to the absorption column. 
In this area, the calculated heat duties were compared by others with various amine solutions with different concentrations, in terms of MEA, DEA, and MEA-MDEA, MEA-AMP [5]. Idem et. al. [4] studied the heat duty of MEA and MEA - MDEA solutions with the small-scale process, which indicated the blended amine solutions costs much less energy than single amine solutions. Zhang et al. [10] performed amine regeneration with different thermomophic biphasic amine solvents. Galindo et al. [11]studied amine regeneration of MEA and DEA solutions (3.25M, 5M and 6.5 M) with a self-designed lab-scale stripper under $70-90^{\circ} \mathrm{C}$ with different reboiler temperatures 110 $130^{\circ} \mathrm{C}$. Li et al. [12] Studied the heat duty of MEA and MEA/MDEA solutions to detect the sensitivity of heat to operation parameters. Zhang et al. [13] have developed experimental studies of heat duty of $\mathrm{CO}_{2}$ desorption from $\mathrm{R}_{3} \mathrm{~N}$ : Ditheylenetriamine(DETA), and $\mathrm{Xu}$ et al. [14] studied regeneration of another $\mathrm{R}_{3} \mathrm{~N}$ : Diethylethanolamine(DEEA). These researches focused on parameters affecting the regeneration energies, such as amine concentration, rich amine loading and flowrate, feeding temperature, reboiler temperature and stripping pressure [11,12]. However, the relationship of energy cost with intrinsic chemical reactions inside amine solutions were not emphasized [11,12].

The research scope was narrowed down to single amine solutions without the parameter optimization of process intensification of any $\mathrm{CO}_{2}$ desorber. This work did not involve any catalysts or blended amine solutions. A conventional solvent recirculation process was adopted to simplify the regeneration. MEA, MMEA and DEA solutions generating carbamate during $\mathrm{CO}_{2}$ absorption were investigated. MEA has been widely used as fundamental chemicals for $\mathrm{CO}_{2}$ desorption. DEA and MMEA are typical secondary amines. Tertiary amines were not studied because of no carbamate.

This research focused on the relative energy cost: in terms of intrinsic reactions, specific $\mathrm{CO}_{2}$ loadings, $\mathrm{CO}_{2}$ related anions and thermodynamics during the regeneration process. The fast / slow stages were proposed for amine regeneration with MEA and MEA-MDEA solutions before [11], the details of turning points (specific $\mathrm{CO}_{2}$ loadings) that distinguish the fast and slow amine regenerations regions were limited due to inadequate experimental data. These regeneration curves contained 4 dots per curve only [15].

Finally, this research objective are: 1) the amine regeneration curves, the "turning point (specific $\mathrm{CO}_{2}$ loadings)" which distinguish different regeneration stages and time periods. 2) The relative heat duty were measured for the first 2 hours of desorption. The order of heat duty is MMEA > MEA > > DEA.

\section{Chemicals and Experimental Procedures}

\section{Chemicals}

The $\mathrm{CO}_{2}$ gas was purchased from Tansool Chemical Ltd, and the chemicals of amines, such as MEA, DEA, and MMEA were purchased from Guoyao Chemical Ltd. The HCl and methyl orange are commercial available from Klamar Chemical Ltd. for $\mathrm{CO}_{2}$ loading tests.

\section{$\mathrm{CO}_{2}$ Desorption / Amine Regeneration Apparatus}

A set of heating-recirculation process was built (Fig 1). The stirrer \& heater was placed at the bottom, with a $1000 \mathrm{ml}$ three-neck bottle inside. The amine solutions with different concentrations 1 , 3,5 , and $7 \mathrm{~mol} / \mathrm{L}$ were placed in the bottle with magnetic stir inside. A thermometer was placed into the amine solution through one side neck to detect the temperature. The temperatures are maintained within the range of $90-105^{\circ} \mathrm{C}$. The other side neck was sealed with a glass cock, ready for the sampling at the appropriate time. The $\mathrm{CO}_{2}$ loading tests of the samples were performed separately with a Chittick apparatus (AOAC), with an AAD of 2.5\% [15]. A condenser was placed on the center neck of the bottle to condense the vapor with recycling water. 


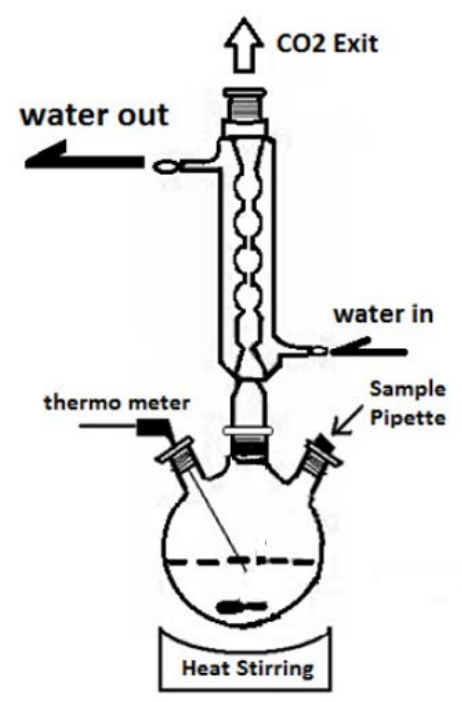

Figure 1. Recirculation process for amine regeneration experiments.

\section{Experimental Operations and Procedures}

The amine regeneration tests were performed with a recirculation process under heating. The amine solution were prepared at $460 \mathrm{ml}$ for 1,3 , and $5 \mathrm{M}$ series for all the amines, but $330 \mathrm{ml}$ for $7 \mathrm{M}$ solutions. The $5 \mathrm{M}$ and $7 \mathrm{M}$ amine solution contains same amount of amine $(\sim 2.3 \mathrm{~mol})$ to analyze the effect of amine concentration toward energy cost. Different amines with same concentrations were categorized into same group for the data analyses.

From Fig 1, the amine solutions were pre-loaded with adequate $\mathrm{CO}_{2}$. The initial loading should be higher than the theoretical loading of $0.50 \mathrm{~mol} / \mathrm{mol}$ to ensure almost all the free amines are converted [11]. The solution was placed into the three-neck bottle and heated with stirring. The condensed water was introduced into the condenser right after heating. These vapors are refluxed back to the flask to maintain the solvent concentration [11]. The condenser was necessary to avoid the interference of water vapours, since the water and solvent vapours need to be cool down while carried over with the exit gas [11]. After the temperature of the solution reached $95^{\circ} \mathrm{C}$, the time of the regeneration process was recorded and the sampling process started. During each regeneration process, the samples were pipetted at $2 \mathrm{ml}$ every $5 \mathrm{~min}$ for the first 0.5 hour, and every $15 \mathrm{~min}$ for the rest 1.5 hours. After the first 2 hours, the rest samples were pipetted every 30 min until 8-9 hours.

In conclusion, the overall regeneration process took 8 - 9 hours, along with 20 - 28 samples collected. The $\mathrm{CO}_{2}$ loading of the samples were tested separately.

\section{Results and Discussion}

\section{The Characteristics of Amine Regeneration Curves and the Turning Points}

Fig. 2-5 demonstrated the amine regeneration curves of MEA, MMEA and DEA at $1 \mathrm{M}, 3 \mathrm{M} 5 \mathrm{M}$ and $7 \mathrm{M} . \mathrm{CO}_{2}$ loadings vs time (h) were plotted properly. The $\mathrm{CO}_{2}$ loading strongly influence the regeneration energy and should be the highest for the absorber to achieve $(>0.5 \mathrm{~mol} \mathrm{CO} / \mathrm{mol}$ amine) [11]. The extra loading over $0.50 \mathrm{~mol} / \mathrm{mol}$ were considered as bicarbonate in water, which is very easy to desorb at the beginning of heating [11]. Finally, the regeneration tests of MEA and MMEA solutions end at lean loading of $0.20 \mathrm{~mol} / \mathrm{mol}$, which is the conventional operation region of amine regeneration of MEA [5]. The regeneration of DEA solutions ends at a selected lean loading of $0.10 \mathrm{~mol} / \mathrm{mol}$, because Galindo discovered DEA reaches an outstanding low loading of $0.10 \mathrm{~mol} / \mathrm{mol}$ at its optimum with low energy cost [11]. 


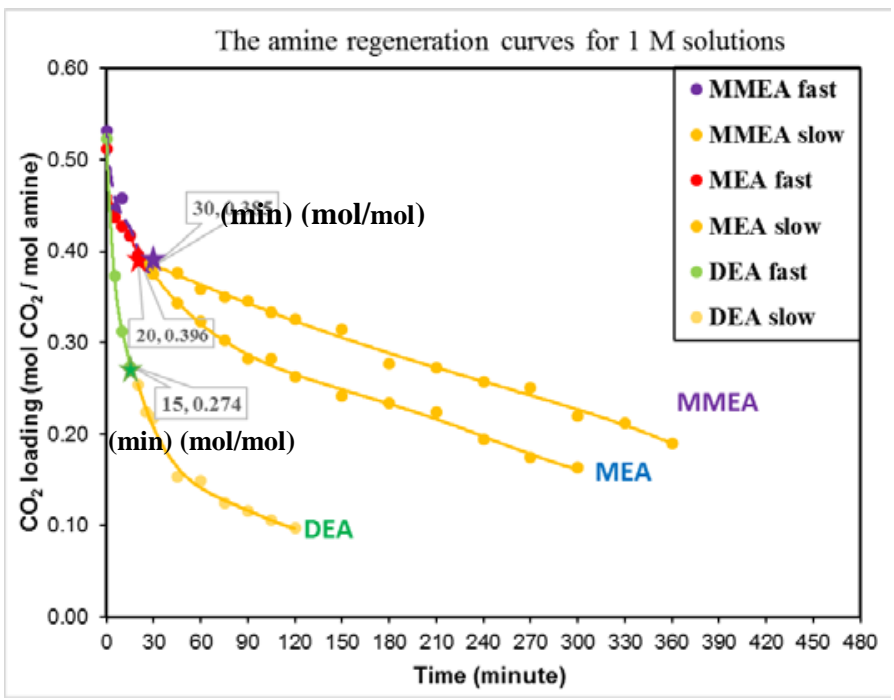

$1 \mathrm{M}$ amine solutions, the turning point is: $0.396 \mathrm{~mol} \mathrm{CO}_{2} / \mathrm{mol}$ for MEA, $0.385 \mathrm{~mol} \mathrm{CO} / \mathrm{mol}$ for MMEA, and $0.274 \mathrm{~mol} \mathrm{CO}_{2} / \mathrm{mol}$ for DEA.

Fig. 2 The primary amine regeneration curves of $460 \mathrm{ml} 1 \mathrm{M}$ MEA, MMEA and DEA solutions at $95-100^{\circ} \mathrm{C}$.

From these series of regeneration curves, the regeneration curves can be split into two stages, the slow stage after the fast stage, with respect to different slopes of the curves $[15,16,17]$. The $\mathrm{CO}_{2}$ desorption rate is relatively high at fast stage. From the start $\mathrm{CO}_{2}$ loading $>0.50 \mathrm{~mol} / \mathrm{mol}$, it decrease 0.1-0.15 mol/mol at the first 15-25 minutes for MEA and MMEA solutions. It decrease $0.20-0.25 \mathrm{~mol} / \mathrm{mol}$ at first $15-20$ minutes for DEA solutions. On the other hand, the rate is apparent low of amine regenerations at slow stage, sometimes it only decrease $0.005-0.03 \mathrm{~mol} \mathrm{CO} / \mathrm{mol}$ amine within one hour. Slow stage is quite energy consuming and less energy efficient.

The turning points were labelled on Fig. 2 - 5. It was surprised to discover that the same amines has similar turning point (specific $\mathrm{CO}_{2}$ loading). The turning point of MEA solutions are at 0.395$0.400 \mathrm{~mol} / \mathrm{mol}$, and that of MMEA and DEA are at $0.380-0.385 \mathrm{~mol} / \mathrm{mol}$ and $0.274-0.280 \mathrm{~mol} / \mathrm{mol}$ at 1-7 M. These turning points were within the region of $\pm 0.0025 \mathrm{~mol} / \mathrm{mol}$ around the center. They turned out to be determined by the chemical property of the amine but not the concentrations.

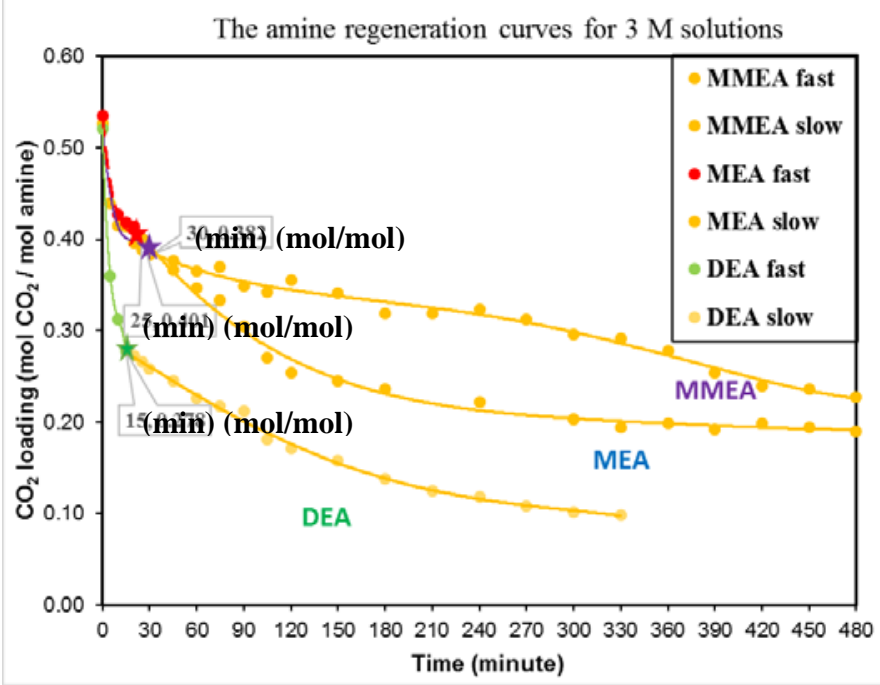

3M amine solutions, the turning point is: $0.401 \mathrm{~mol} \mathrm{CO}_{2} / \mathrm{mol} \mathrm{MEA} 0.382 \mathrm{~mol} \mathrm{CO}_{2} / \mathrm{mol} \mathrm{MMEA}$, and $0.278 \mathrm{~mol} \mathrm{CO}_{2} / \mathrm{mol}$ DEA.

Fig. 3 The primary amine regeneration curves of $460 \mathrm{ml}$ 3M MEA, MMEA and DEA solutions at $95-100{ }^{\circ} \mathrm{C}$. 


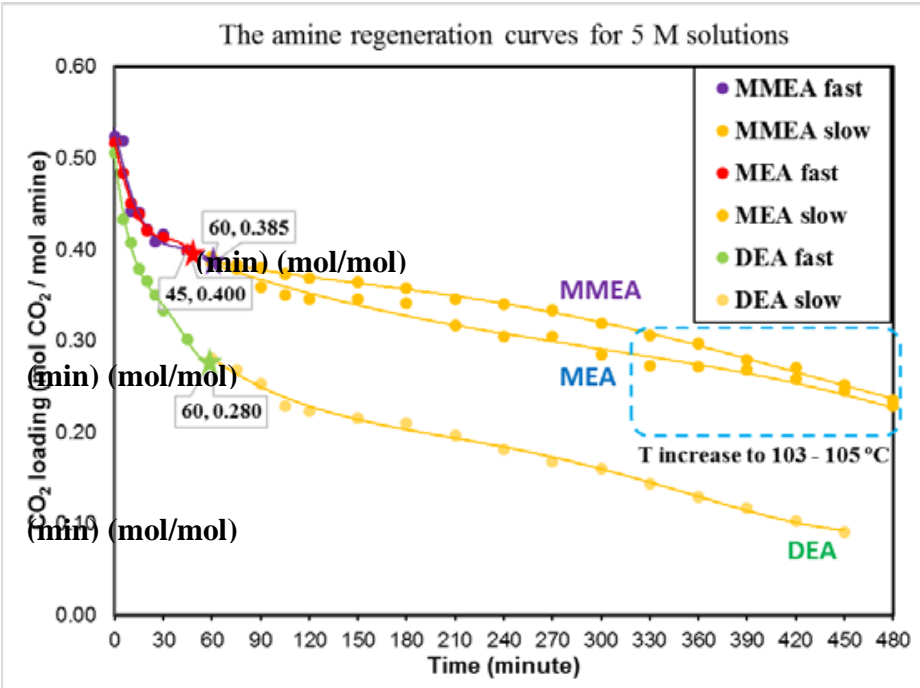

$5 \mathrm{M}$ amine solutions: the turning point is $0.400 \mathrm{~mol} \mathrm{CO}_{2} / \mathrm{mol}$ of MEA, $0.385 \mathrm{~mol} \mathrm{CO} / \mathrm{mol}$ of MMEA, and $0.280 \mathrm{~mol} \mathrm{CO}_{2} / \mathrm{mol}$ amine of DEA.

Fig. 4 The primary amine regeneration curves of $460 \mathrm{ml}$ 5M MEA, DEA and MMEA solutions at $95-105^{\circ} \mathrm{C}$.

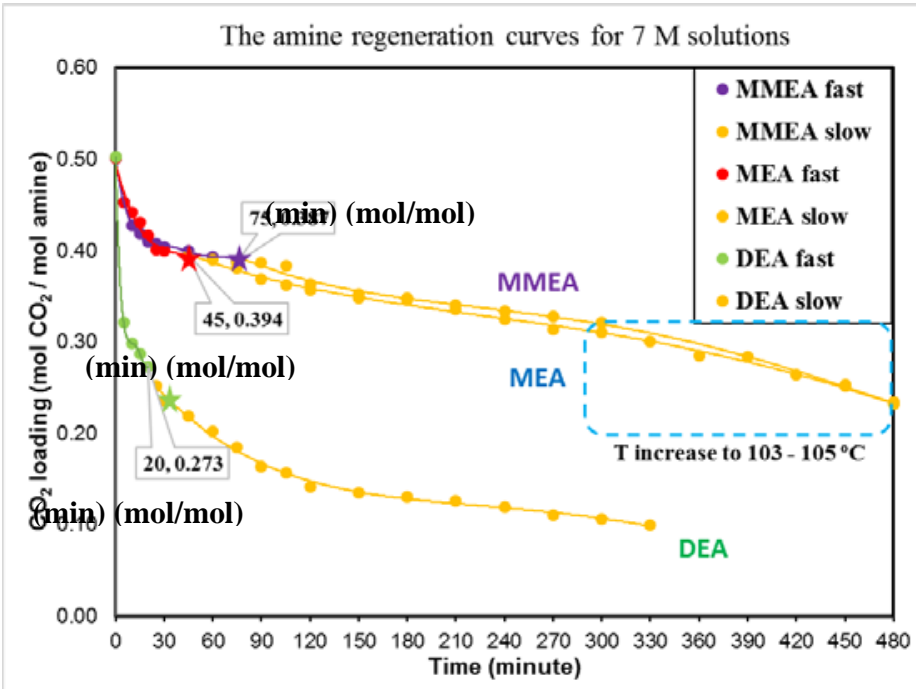

$7 \mathrm{M}$ amine solutions: the turning point is $0.400 \mathrm{~mol} \mathrm{CO}_{2} / \mathrm{mol}$ of MEA, $0.387 \mathrm{~mol} \mathrm{CO} 2 / \mathrm{mol}$ of MMEA and $0.280 \mathrm{~mol} \mathrm{CO}_{2} / \mathrm{mol}$ amine of DEA.

Fig. 5 The primary amine regeneration curves of $330 \mathrm{ml}$ 7M MEA and DEA solutions at $95-105{ }^{\circ} \mathrm{C}$.

\section{The Relative Heat Duty Analyses for the First 2 Hours}

The operation region of MEA regeneration was 0.50-0.20 mol/mol [5], and DEA can reach leaner loading at $0.10 \mathrm{~mol} / \mathrm{mol}$ [5] under the similar reboiler heat duty [11]. The relative heat duty was also the research focus, and the absolute energy cost require an electric energy meter to measure electric power of the heater [18]. Based on the Figure 2-5 generated, the relative heat duties of these systems were calculated and grouped on Figure 6. It is clear from the figure that higher heat duty can release a larger amount of $\mathrm{CO}_{2}$ product, and left a leaner solution exit the regeneration column [5]. 
If the operation process were performed in a lab-scale desorber with a reboiler, the heat input $\mathrm{Q}$ were calculated with such equation(1) below [5]; where $\dot{m}_{f}, C_{P, f}, t_{i n, f}$ and $t_{o u t, f}$ denote the mass flow rate, heat capacity of heating fluid, temperature of heating fluid entering and exit the reboiler.

$$
\dot{Q}_{\text {input }}=\dot{H}_{\text {reboiler }}=\dot{m}_{f} C_{P, f}\left(t_{\text {in,f }}-t_{\text {out }, f}\right) \text {. }
$$

Unfortunately, the regeneration process did not equip any electrometer to measure the exact energy of heat input. All the energy calculations are the relative value comparing to MEA. The MEA at $1 \mathrm{M}, 3 \mathrm{M}, 5 \mathrm{M}$ and $7 \mathrm{M}$ were regarded as $100 \%$ at its own scenarios, and the relative heat duties were calculated for other amines at the same concentration. Such calculations were made based on the assumptions, that $\mathrm{Q}_{\text {input }}$ were approximately the same under same concentration, since the experiments were conducted with the same process, same operation temperature and same volume of amine solutions.

Therefore, the relative heat duty $\mathrm{H}_{\mathrm{CO} 2}$ were conducted from equation (2) [5], amount of $\mathrm{CO}_{2}$ generation is calculated from equation (2). The results were plotted in Figure 6.

$$
\mathrm{H}_{\mathrm{CO} 2}=\frac{Q_{\text {input }}}{n_{\mathrm{CO} 2}} \frac{k J / \text { hour }}{\text { mol/hour }}
$$

From Figure 6, it is apparent that the relative heat duties of MMEA is bigger than that of MEA at ratios of $137 \%(1 \mathrm{M})$ to $146 \%(3 \mathrm{M}), 118 \%(5 \mathrm{M})$, and $123 \%(7 \mathrm{M})$; and that of DEA is lower than MEA at ratios of $59 \%(1 \mathrm{M}), 70 \%(3 \mathrm{M}), 56 \%(5 \mathrm{M})$ and $40 \%(7 \mathrm{M})$, respectively. The results indicated MMEA as a poor solution for $\mathrm{CO}_{2}$ desorption, but DEA as a good one. These results partly validated peer researchers conclusion: DEA reaches a more effective $\mathrm{CO}_{2}$ desorption process, with lower energy cost, low loading of $0.10 \mathrm{~mol} / \mathrm{mol}$ and higher removal efficiency [11]. DEA is also less reactive to sulphur components and more resistant to corrosion than MEA [11], which makes itself a good candidate of $\mathrm{CO}_{2}$ desorption.

However, DEA exhibits much slower kinetics than that MEA, which was proved by us and peer researchers [11]. Considering the advantages and disadvantages of DEA, the MEA-DEA blended amine with different concentrations may be helpful for amine regeneration and gas purification [19].The MEA can be the main amine with high concentration 3-5 M, and DEA can blend with MEA as subsidy with relative low concentration 1-2 $\mathrm{M}$.

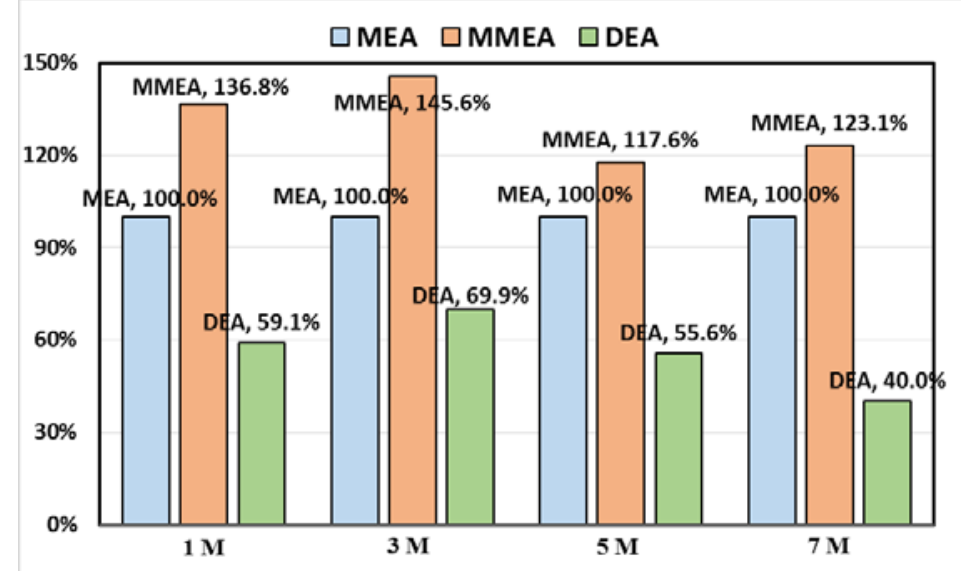

Fig. 6 The relative heat duty of the amines at first 2 hours.

\section{Conclusion}

(1) The amine regeneration tests were conducted in a recirculation process for three typical amines, MEA, MMEA and DEA. The regeneration curves were divided into two regions: fast and slow. The turning points of two regions were $0.40 \mathrm{~mol} / \mathrm{mol}$ for MEA, and $0.38 \mathrm{~mol} / \mathrm{mol}$ for MMEA, and 0.28 $\mathrm{mol} / \mathrm{mol}$ for DEA despite different amine concentrations. The turning points were determined by the chemical property of the amine. 
(2) The relative heat duties (\%) of different amines were compared at the same amine concentration to estimate the energy efficiency. MEA solutions were regarded as $100 \%$. The $\mathrm{CO}_{2}$ desorption of MMEA is harder than MEA with 18-46 \% extra heat duty. DEA requires relative low energy cost of $40-70 \%$ of MEA.

\author{
Abbreviations \\ MEA Monoethanolamine \\ MMEA N-Methyl monoethanolamine \\ DEA Diethanolamine \\ GREEK LETTER \\ $\alpha=\mathrm{CO}_{2}$ loading, $\mathrm{mol} / \mathrm{mol}$ or $\mathrm{mol} \mathrm{CO}_{2} / \mathrm{mol}$ amine

\section{Acknowledgments} \\ This financial supports are provided by Youth Teachers Support Program (ZZsl15042) and Young \\ Eastern Scholar (QD 2016011). The National Natural Science Foundation of China (NSFC Nos. \\ 21606150) are gratefully acknowledged.
}

\title{
Reference
}

[1] R. Idem, T. Supap, H. Shi, D. Gelowitz, M. Ball, C. Campbell, P. Tontiwachwuthikul, Practical experience in post-combustion CO2capture using reactivesolvents in large pilot and demonstration plant, Int. J. of Greenhouse gas contr., 40(2015) 6-25.

[2] Z. Liang, W. Rongwong, H. Liu, K. Fu, H. Gao, F. Cao, R. Zhang, T. Sema, A. Henni, K. Sumon, D. Nath, D. Gelowitz, W. Srisang, C. Saiwan, A. Benamor, M. Al-Marri, H. Shi, T. Supap, C. Chan, Q. Zhou, M. Abu-Zahra, M. Wilson, W. Olson, R. Idem, P. Tontiwachwuthikul, Recent progress and new developments in post-combustion carbon-capture technology with amine based solvents, Int. J. of Greenhouse gas contr., 40(2015) 26-54.

[3] GT. Rochelle, Amine scrubbing for CO2 capture, Science, 325(2009)1652.

[4] R. Idem, M. Wilson, P. Tontiwachwuthikul, A. Chakma, A. Veawab, A. Aroonwilas, D. Gelowitz, Pilot Plant Studies of the CO2 Capture Performance of Aqueous MEA and Mixed MEA/MDEA Solvents at the University of Regina CO2 Capture Technology Development Plant and the Boundary Dam CO2 Capture Demonstration Plant, Ind. Eng. Chem. Res., 45(2006)24142420 .

[5] R. Sakwattanapong, A. Aroonwilas, A. Veawab, Behavior of Reboiler Heat Duty for CO2 Capture Plants Using Regenerable Single and Blended Alkanolamines, Ind. Eng. Chem. Res., 44(2005)4465-4473.

[6] T. Li, TC Keener, A review: Desorption of CO2 from rich solutions in chemical absorption processes, Int. J. Greenhouse Gas Contr., 51(2016)290-304.

[7] A. Aroonwilas, A. Veawab, Integration of $\mathrm{CO} 2$ capture unit using single- and blended-amines into supercritical coal-fired power plants: Implications for emission and energy management, Int. J. Greenhouse Gas Contr., 1(2007)143-150.

[8] H. Gao, L. Zhou, ZW. Liang, R. Idem, K. Fu, T. Sema, P. Tontiwachwuthikul, Comparative studies of heat duty and total equivalent work of a new heat pump distillation with split flow process, conventional split flow process, and conventional baseline process for $\mathrm{CO} 2$ capture using monoethanolamine, Int. J. Greenhouse Gas Contr., 24(2014)87-97. 
[9] US. 8388737 B2 (2013), invs.: D. Gelowitz, R. Idem, P. Tontiwachwuthikul.

[10]J. Zhang, Y. Qiao, D.W, Agar, Intensification of low temperature thermomorphic biphasic amine solvent regeneration for CO2 capture, Chem. Eng. Res. Des., 90 (2012)743-749.

[11]P. Galindo, A. Shaffer, K. Brechtel, S. Unterberger, G. Scheffknecht, Experimental research on the performance of CO2-loaded solutions of MEA and DEA at regeneration conditions, Fuel, 101(2012)2-8.

[12]X. Li, S. Wang, C. Chen, Experimental study of energy requirement of CO2 desorption from rich solvent, Energy Procedia. 27(2013)1836-1843.

[13]X. Zhang, K. Fu, Z. Liang, W. Rongwong, Z. Yang, R. Idem, P. Tontiwachwuthikul, Experimental studies of regeneration heat duty for CO2 desorption from diethylenetriamine (DETA) solution in a stripper column packed with Dixon ring random packing, Fuel, 136(2014)261-267.

[14]B. Xu, H. Gao, M. Chen, Z. Liang, R. Idem, Experimental Study of Regeneration Performance of Aqueous N,N-Diethylethanolamine Solution in a Column Packed with Dixon Ring Random Packing, Ind. Eng. Chem. Res., 55 (2016)8519-8526.

[15]H. Shi, A. Naami, R. Idem, P. Tontiwachwuthikul, Catalytic and non catalytic solvent regeneration during absorption-based $\mathrm{CO} 2$ capture with single and blended reactive amine solvents, Int. J. Greenhouse Gas Contr., 26(2014)39-50.

[16]Z. Yu, H. Shi, Effective energy efficient methods for heat duty reduction for amine-based postcombustion capture process based on the theoretical reactions energy calculation, Int. J. Oil, Gas and Coal Technology, 14(2017)172.

[17]H. Shi, R. Idem, A. Naami, D. Gelowitz, P. Tontiwachwuthikul, Catalytic Solvent Regeneration Using Hot Water During Amine Based CO2 Capture Process, Energy Procedia, 63(2014)266-272.

[18]Z. Liang, R. Idem, P. Tontiwachwuthikul, F. Yu, H. Liu, W. Rongwong, Experimental study on the solvent regeneration of a CO2 - loaded MEA solution using single and hybrid solid acid catalysts, AICHE J, 62(2015)753.

[19]A.L. Kohl, R.B. Nielsen, Gas Purification, 5th edition, Gulf Publishing Co., Houston, TX 1997. 